\title{
Detecting masked high blood pressure in high-risk patients
}

\author{
Hyungseop Kim
}

Division of Cardiology, Department of Internal Medicine, Keimyung University Dongsan Medical Center, Daegu, Korea
Received: July 20, 2015

Accepted: July 24, 2015

\section{Correspondence to}

\section{Hyungseop Kim, M.D.}

Division of Cardiology,

Department of Internal Medicine,

Keimyung University Dongsan

Medical Center, 56 Dalseong-ro,

Jung-gu, Daegu 41931, Korea

Tel: $+82-53-250-7427$

Fax: +82-53-250-7034

E-mail: khyungseop@dsmc.or.kr

\section{See Article on Page 610-619}

High blood pressure (BP), or hypertension, is a risk factor for cardiovascular (CV) disease. Unfortunately, BP is not always constant throughout life, being easily affected by physical condition, mental stress, environmental factors, infection, hormonal balance, and many variables that modulate the activities of the autonomic nervous and renin/angiotensin systems. Thus, hypertension is not easy to diagnose; $\mathrm{BP}$ cutoffs triggering timely antihypertensive treatment are under constant revision and updating. Ambulatory blood pressure monitoring (ABPM) is recommended to clearly define and evaluate hypertension; clinical BP measurements are inadequate [1].

In terms of BP fluctuations, the frequency of BP measurement is critically important because a clinical BP is the average of only three to five measurements. One or two clinical BP measurements cannot detect patients requiring long-term antihypertensive treatment. Although whether ambulatory or clinical BP measurement is superior remains controversial, the advantages of ABPM have become gradually recognized in recent epidemiological studies. ABPM can also be used to diagnose masked or white-coat hypertension [2-4]. In the present issue of the Journal,
Shin et al. [5] address the importance of ABPM from a clinical viewpoint and show that risk stratification can be used to identify those who both need and can benefit from ABPM. Even in treated well-controlled patients, it is important to explore the presence of masked sustained hypertension. The cited authors found that reductions in white-coat hypertension were associated with less disagreement between BP measured clinically and via ABPM in very high-risk patients. The prevalence of persistently high masked hypertension was $>10 \%$. Therefore, uncontrolled masked hypertension is a serious issue even in treated patients at high and very high added risk.

The clinical risk factors for the development of masked hypertension are generally similar to those for the development of sustained hypertension, and the prognoses of masked hypertension are unfavorable in both untreated and treated groups [6]. This may be attributable to (inappropriate) under-treatment of hypertension. Notably, almost $60 \%$ of well-controlled hypertensive patients were re-classified as exhibiting masked hypertension, and 10\% had white-coat hypertension [7]. The recently established Spanish ABPM registry found that $A B P M$ was essential for appropriate management of hypertension, and could be used to diagnose, and discriminate between, white-coat 
and truly resistant hypertension [2]. ABPM re-classified > $30 \%$ of resistant hypertension patients as cases of whitecoat hypertension (as evidenced clinically). Patients with truly resistant hypertension had higher frequencies of poor CV risk profiles (smoking, diabetes, and target organ damage). Furthermore, masked, rather than whitecoat, hypertension was associated with atherogenesis, atherosclerosis, and vascular inflammation in the highrisk group [8]. Such patients exhibited a tendency toward the development of atherosclerosis, including increased thickness of the carotid intima media, compared with the low-risk group [9]. When the treatment of masked hypertension is considered, the validity of clinical BP-based treatment must be questioned. It remains unclear whether such treatment actually mitigates hypertension-mediated organ damage.

However, ABPM can cause stress and inconvenience; the technique is not easy to apply. Should we use ABPM to screen or monitor treatment responses in all hypertensive patients? Firm evidence that ABPM improves clinical outcomes in hypertensive patients is lacking. Thus, ABPM is currently performed on limited numbers of patients. Previous studies have emphasized that much effort is required to reduce $\mathrm{CV}$ events in patients with masked hypertension and to optimize the treatment of white-coat hypertension; ABPM is essential in these contexts $[2,5,10]$. When detecting or evaluating hypertension, the first step is usually clinical BP measurement, which cannot adequately detect hypertension because nocturnal high BP is not evaluated. Generally, ABPM is considered when patients exhibit clinical evidence of high-level CV risk factors or complicated organ damage. Similarly, Shin et al. [5] explored when ABPM was useful in hypertensive patients and found that, in patients with a more than moderate CV risk, ABPM (helpfully) revealed masked hypertension. Also, ABPM played a key role in monitoring responses to treatment, particularly in high-risk patients. In addition, the cited authors explained when ABPM should be used to detect white-coat hypertension in treated moderate-risk patients. Notably, in treated high- or very high-risk patients, the BP control rate was somewhat poorer than in other groups; treated subjects remained uncontrolled (thus with sustained hypertension). Therefore, physicians should always determine whether BP control is effective in high-risk patients.
One of the greatest advantages of ABPM compared to clinical BP assessment is that ABPM can predict organ damage associated with high $\mathrm{BP}$ and allows the stratification of hypertensive patients by the risk of $\mathrm{CV}$ events. This may explain why ABPM is better than clinical BP measurement in high-risk patients. Conversely, it seems likely that clinical BP measurements do not allow adequate evaluation of high-risk patients because such measures do not detect complications associated with high BP. Such complications are frequently evident in high- or very high-risk patients. Therefore, it is reasonable to identify and treat "sustained or masked hypertension" rather than "white-coat hypertension" in high-risk groups.

In brief, although the study has some limitations, including the absence of data on adherence to medication regimes, ABPM is essential to evaluate the responses of, and to discriminate between, high-risk patients with masked and white-coat hypertension for whom intensive appropriate treatment is essential. However, additional studies are needed to support the widespread use of ABPM. The link between CV risk and the BP pattern over a 24-hour period requires further study.

\section{Conflict of interest}

No potential conflict of interest relevant to this article was reported.

\section{REFERENCES}

1. Mancia G, Fagard R, Narkiewicz K, et al. 2013 ESH/ ESC Guidelines for the management of arterial hypertension: the Task Force for the management of arterial hypertension of the European Society of Hypertension (ESH) and of the European Society of Cardiology (ESC). J Hypertens 2013;31:1281-1357.

2. de la Sierra A, Segura J, Banegas JR, et al. Clinical features of 8295 patients with resistant hypertension classified on the basis of ambulatory blood pressure monitoring. Hypertension 2011;57:898-902.

3. Parati G, Stergiou G, O’Brien E, et al. European Society of Hypertension practice guidelines for ambulatory blood pressure monitoring. J Hypertens 2014;32:1359-1366.

4. Conen D, Aeschbacher S, Thijs L, et al. Age-specific differences between conventional and ambulatory daytime 
blood pressure values. Hypertension 2014;64:1073-1079.

5. Shin J, Park SH, Kim JH, et al. Discordance between ambulatory versus clinic blood pressure according to global cardiovascular risk group. Korean J Intern Med 2015;30:610-619.

6. Stergiou GS, Asayama K, Thijs L, et al. Prognosis of whitecoat and masked hypertension: international database of home blood pressure in relation to cardiovascular outcome. Hypertension 2014;63:675-682.

7. Lehmann MV, Zeymer U, Dechend R, et al. Ambulatory blood pressure monitoring: is it mandatory for blood pressure control in treated hypertensive patients?: prospective observational study. Int J Cardiol 2013;168:22552263.
8. Balta S, Demirkol S, Celik T, Unlu M, Kucuk U, Arslan Z. Inflammatory markers should be assessed together with cardiovascular risk factors by clinicians in masked hypertension. J Clin Hypertens (Greenwich) 2013;15:443444 .

9. Hara A, Ohkubo T, Kikuya M, et al. Detection of carotid atherosclerosis in individuals with masked hypertension and white-coat hypertension by self-measured blood pressure at home: the Ohasama study. J Hypertens 2007;25:321-327.

10. Banegas JR, Ruilope LM, de la Sierra A, et al. High prevalence of masked uncontrolled hypertension in people with treated hypertension. Eur Heart J 2014;35:3304-3312. 